\title{
FATORES DA EMBALAGEM DE LEITE COM CONCENTRAÇÃO AUMENTADA DE MELATONINA NA INTENÇÃO DE COMPRA DO CONSUMIDOR
}

\section{Milk with increased concentration of melatonin packaging factors on consumer purchase intention}

\author{
Maria Patricia Milagres ${ }^{1 *}$, Valéria Paula Rodrigues Minim², Andréa Alves Simiqueli², \\ Ana Cristina Rocha Espeschit ${ }^{2}$, Luis Antonio Minim ${ }^{2}$
}

\section{RESUMO}

A análise conjunta de fatores foi empregada para desenvolver um rótulo para a embalagem do leite com concentração aumentada de melatonina. Foram selecionados três fatores, com três ou dois níveis para a montagem das embalagens: design da embalagem ("azul com estrelas e desenho de leite derramando", "branca sem desenho" e "azul com estrelas e sem desenho"), nome ("leite noturno" e "melatonina") e texto informativo sobre obtenção natural de melatonina por meio de ordenha noturna, e os benefícios deste composto ("com" e "sem"). Doze imagens de embalagens foram criadas baseando-se em um arranjo fatorial completo. Estas imagens foram apresentadas a 144 consumidores, aos quais foi solicitado que avaliassem a intenção de compra de cada embalagem. Pela análise conjunta de fatores, o produto ideal ou de maior probabilidade de compra para a maioria dos consumidores seria a embalagem azul com estrelas e desenho de leite derramando, com o nome leite noturno e com a presença de texto informativo. Desta forma, demonstrou-se que os consumidores gostam de uma embalagem que remete o produto e que contenha informações descritas. Logo, a embalagem apresenta grande influência na escolha do consumidor, podendo contribuir de forma positiva ou negativa para a aceitação do alimento.

Palavras-chave: análise conjunta de fatores; sono; leite com melatonina.

1 Universidade Estadual do Sudoeste da Bahia (UESB), Departamento de Quimica e Exatas, Curso de Farmácia, Estrada do Bem Querer, km 04, Zona Rural, 45083-900, Vitória da Conquista, BA, Brasil. E-mail: patricia@uesb.edu.br.

2 Universidade Federal de Viçosa (UFV), Departamento de Tecnologia de Alimentos, Viçosa, MG, Brasil.

* Autor para correspondência 


\begin{abstract}
The conjoint analysis of factors was used employed to develop a label for the packaging of milk with increased concentration of melatonin. Three factors were selected with three or two levels for the assembly of packaging: packaging design ("blue design with stars and pouring milk", "white without drawing" and "blue stars with and without drawing"), name ("milk night "and" melatonin ") and informative text about getting natural melatonin by night milking, and the benefits of this compound ("with" and "without"). Twelve packaging designs were created based on a complete factorial arrangement. The designs were presented to 144 consumers who were asked to assess purchase intent of each package. For the joint analysis of factors, the ideal product or more likely to purchase for the most consumers would be blue with stars and packaging design of milk pouring, named milk night and in the presence of informational text, demonstrating that consumers like a package that brings the product and that contains information described. Therefore, the packaging has a major influence on consumer choice and can contribute positively or negatively to the acceptance of food.
\end{abstract}

Keywords: conjoint analysis of factors; consumer; packaging; milk with melatonina.

\section{INTRODUÇÃO}

A melatonina é responsável pela regulação circadiana e pelo controle do sono, sendo encontrada em maior concentração no organismo durante a noite. Distúrbio em sua produção pode ser responsável pela insônia. Uma queda substancial na produção de melatonina acontece com a idade, o que pode prejudicar a qualidade do sono dos idosos. Desta forma, é importante a observação e a regulação da produção da melatonina no organismo, uma vez que a insônia e os distúrbios do sono causam diversos malefícios à saúde e à produtividade das pessoas. Uma forma de aumentar a melatonina no organismo é por meio da alimentação, uma vez que o composto é encontrado em diversos alimentos como mostarda, erva-doce e leite. Porém, nestes alimentos a melatonina se encontra em baixas concentrações e o seu aumento, pela adição de melatonina no alimento, não é permitido pela legislação (MILAGRES, 2012). Assim, diversos trabalhos têm sido desenvolvidos objetivando a obtenção de leite com concentração de melatonina aumentada por meio das ordenhas noturnas (VALTONEN et al., 2001) ou ordenhas com condições controladas de iluminação (HAIGH, 2003).

Apesar de serem encontrados leites com concentração aumentada de melatonina no mercado, nenhum estudo foi realizado com objetivo de definir os fatores da embalagem. Esses são considerados importantes para os consumidores, e assim, é possível desenvolver uma embalagem atrativa para o produto inovador.

Além da qualidade sensorial de alimentos, outros fatores estão relacionados com a formação de expectativas e com o processo de seleção de um produto (GADIOLI et al., 2013).

A embalagem tem importância fundamental na escolha do produto durante a compra, uma vez que ela representa o primeiro contato entre o consumidor e o produto. Fatores relacionados à embalagem como cor, textura e informações influenciam na intenção 
de compra dos consumidores. Assim, para otimizar o processo de escolha e aceitação do produto, é necessário identificar os fatores da embalagem valorizados pelo consumidor (DELLA LUCIA et al., 2007).

Estudos de Costa (1999), Carneiro et al. (2005), Della Lucia et al. (2007), Souza et al. (2007), Ferrarezi (2008), Frata et al. (2009), Gonçalves (2009), Gadioli (2013) e Mesias (2013) mostram que grande parte dos fatores considerados importantes para o consumidor podem ser observados na embalagem do produto.

Visto o importante papel que a embalagem desempenha em atrair a atenção dos consumidores influenciando a percepção do produto e suas decisões de compra,é importante encontrar metodologias que permitem determinar com precisão a importância da embalagem no processo de compra e os formatos mais preferidos. Entre os diferentes métodos que podem ser utilizados para este fim, a Análise Conjunta de Fatores é um dos mais completos. Ele permite avaliar a importância relativa dos diferentes atributos que constituem a estrutura das preferências dos consumidores. Devido ao seu potencial, tem sido amplamente utilizada no setor agro-alimentar (MESIAS et al., 2013).

Uma característica comum nos estudos com embalagem de produtos alimentícios é a quantificação do efeito que cada característica presente nela tem sobre a intenção de compra do consumidor. A análise conjunta de fatores vem a ser uma técnica utilizada, com êxito, para este fim. Esta técnica, quando empregada juntamente com métodos multivariados, permite entender como os indivíduos desenvolvem preferência por produtos ou serviços, baseando-se em suas diferentes características. Seu emprego gera a base para se descrever a relação do consumidor com o produto e possibilita, com isso, o aprimoramento das estratégias de marketing da indústria (DELLA LUCIA et al., 2007).
No caso de alimentos funcionais, que apresentam algum beneficio à saúde, como por exemplo, os que contêm melatonina, composto que irá favorecer na qualidade do sono (KENNAWAY; WRIGHT, 2002), a importância da embalagem parece ser ainda maior. Pois, ela deve transmitir informações que levem o consumidor a ter segurança de estar comprando um produto que faça bem à sua saúde. Neste contexto, objetivou-se desenvolver um rótulo para a embalagem para o leite com concentração aumentada de melatonina, por meio da Análise Conjunta de Fatores.

\section{MATERIAL E MÉTODOS}

O trabalho foi realizado utilizando a Análise Conjunta de Fatores segundo Minim (2010).

\section{Escolha dos fatores da embalagem}

\section{O Sleep Disorders Questionnaire} (SWEERE et al., 1998) adaptado para a forma estruturada com questões socioeconômicas e demográficas foi usado como questionário de recrutamento. Foram selecionadas pessoas que apresentavam problemas de sono e tinham interesse em participar do trabalho.

As pessoas recrutadas participaram de sessões de Grupo de Focus, realizado segundo Della Lucia; Minim (2010).

Cinco sessões de grupo de focos foram conduzidas com um total de 37 participantes. As sessões foram realizadas na sala de reuniões no Departamento de Tecnologia de Alimentos (UFV): a primeira sessão contou com a participação de 7 voluntários (5 mulheres e 2 homens); a segunda, com 8 (4 mulheres e 4 homens); a terceira, com 6 pessoas (5 mulheres e 1 homem); a quarta, com 7 (4 mulheres e 3 homens); e a quinta, com 9 participantes ( 7 mulheres e 2 homens).

As sessões ocorreram em mesa redonda contando com um moderador e um assistente, 
que foi encarregado de gravar, filmar e anotar as informações de cada sessão. O moderador explicou o propósito da sessão e esclareceu a importância da opinião de cada participante no estudo. Foi utilizado um roteiro (Tabela 1) de perguntas sobre os hábitos dos consumidores durante as suas compras e a apresentação de quatro embalagens de leite de marcas comerciais e uma de produto desenvolvido (protótipo) (Figura 1). Essas embalagens foram apresentadas separadamente e em ordem aleatória. As sessões tiveram duração média de 90 minutos. Também foi ressaltado que não existe resposta certa para as questões abordadas, sendo importante a opinião de cada um.

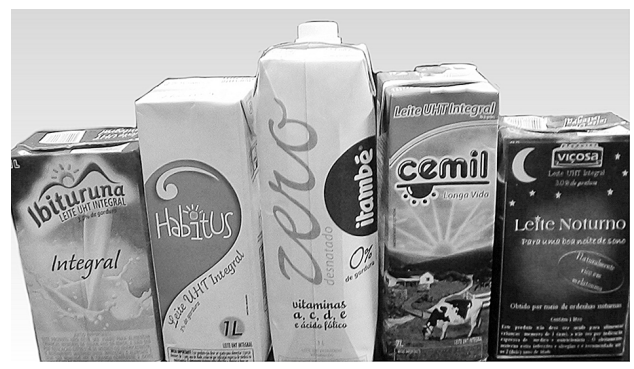

Figura 1 - Embalagens apresentadas nas sessões de grupo de focos.

Os dados obtidos nas gravações e anotações foram usados para a identificação de fatores do rótulo que mais influenciam no processo de escolha do produto pelos consumidores durante as compras em supermercados. A partir daí, foram enumerados os fatores julgados importantes pelos grupos. E para cada um dos fatores escolhidos, foram definidos os seus níveis.

Os fatores nome do produto, texto informativo sobre o leite com concentração aumentada de melatonina, e o design da embalagem foram escolhidos para compor os tratamentos avaliados (Tabela 2). Esses fatores foram selecionados de acordo com as ideias expostas nas sessões de grupo de focos.

\section{Coleta de dados e arranjo experimental}

O método de coleta de dados utilizado neste estudo foi o de perfil completo (GREEN; SRINIVASAN, 1978). Cada tratamento (embalagem) avaliado foi composto pela combinação dos três fatores predefinidos design da embalagem, nome do produto e texto informativo - sendo, assim, constituído de um nível desses fatores.

$\mathrm{O}$ arranjo de tratamentos utilizado foi do tipo fatorial completo, ou seja, o número de tratamentos avaliados correspondeu a todas as possíveis combinações entre os três fatores e cada um de seus níveis. Desse modo, foram

Tabela 1 - Roteiro para as sessões de grupo de focos

1) Você observa os rótulos de produtos que consome?

2) O que você observa?

3) Você conhece um composto denominado melatonina?

4) $\mathrm{O}$ que você acha que seja?

5) Apresentação sobre a melatonina abordando o que é o composto, seus benefícios, os alimentos que têm este produto, a ordenha noturna e o aumento da concentração de melatonina naturalmente no leite.

6) O que você acha do leite com concentração de melatonina aumentada naturalmente por ordenhas noturnas?

7) Você consumiria este produto?

8) O que você achou desta embalagem? Comentar sobre cor, nome e informações. 
obtidos 12 tratamentos $\left(n^{\mathrm{f}}=2^{2} \quad 3^{1}=12\right)$, que estão apresentados na Tabela 3 .

\section{Confecção das embalagens}

As embalagens foram confeccionadas por uma empresa especializada. O design da embalagem foi padronizado para caixa retangular, nas medidas $25,5 \mathrm{~cm} \mathrm{x} 7 \mathrm{~cm} \mathrm{x}$ $6 \mathrm{~cm}$, com tampas rosqueáveis, baseada na opinião dos consumidores que participaram do grupo de focos (Figura 2).

As embalagens foram apresentadas aos voluntários de forma monádica sob luz branca no Laboratório de Análise Sensorial da Universidade Federal de Viçosa. Não foi definido um tempo de julgamento para as embalagens. Em cada avaliação de uma nova embalagem, o consumidor recebeu a ficha de avaliação identificada com um número de três dígitos correspondente ao tratamento em questão.

Foi utilizada para a avaliação da intenção de compra a escala linear horizontal não-estruturada de $9 \mathrm{~cm}$. Essa escala é composta de duas âncoras nas extremidades: na extremidade esquerda está escrita a expressão "definitivamente não compraria" e na direita, "definitivamente compraria".
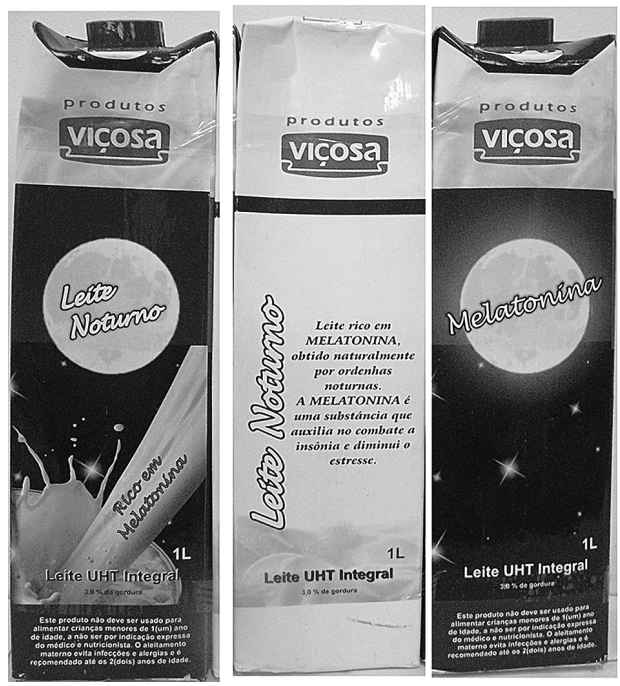

Figura 2 - Exemplos de embalagens avaliadas

A primeira embalagem ap resentada foi igual para todos os consumidores, possuindo um painel frontal sem nenhuma informação acerca dos fatores em estudo. A apresentação dessa embalagem tem como objetivo retirar o

Tabela 2 - Fatores das embalagens e respectivos níveis

\begin{tabular}{ll}
\hline Fatores & Níveis/descrição \\
\hline & $\begin{array}{l}\text { 1-Azul com estrelas e desenho de leite } \\
\text { derramando }\end{array}$ \\
Design da embalagem & $\begin{array}{l}\text { 2-Branca sem desenho } \\
\text { 3-Azul com estrelas e sem desenho }\end{array}$ \\
Nome & $\begin{array}{l}\text { 1- Leite noturno } \\
\text { 2-Melatonina }\end{array}$ \\
Texto informativo & \\
Leite rico em melatonina obtido & 1-Com \\
naturalmente por ordenhas noturnas. & 2-Sem \\
A melatonina é uma substância & \\
que auxilia no combate à insônia & \\
e diminui o estresse & \\
\hline
\end{tabular}


efeito da primeira amostra ou o impacto que o primeiro tratamento apresentado poderia causar sobre a avaliação das demais amostras.

A apresentação das amostras seguiu o delineamento proposto por MacFie et al. (1989), em que cada embalagem aparece em certa posição o mesmo número de vezes. Esse delineamento garante a eliminação do efeito da ordem de apresentação e o efeito residual caracterizado pela influência de uma amostra na avaliação subsequente.

As 48 sequências de apresentação dos tratamentos, de acordo com o delineamento selecionado, são suficientes para estimar os quatro efeitos principais de modo não-viesado. Três repetições do delineamento foram empregadas na avaliação das embalagens, de maneira que 144 consumidores participaram do estudo.

\section{Análise dos dados}

Os resultados foram avaliados conforme proposto por Minim (2010). As respostas das fichas de avaliação da intenção de compra dos consumidores foram transformadas em escores, medindo-se a distância que vai desde a extremidade esquerda até a marca feita pelo consumidor, tendo estes escores variado entre 0 e 9 . Os resultados foram tabulados em forma de escores para cada embalagem avaliada, em um quadro de dupla entrada de consumidores versus embalagens.

Como regra de composição, foi utilizado o modelo aditivo. Esse modelo assume que as avaliações globais de preferência são formadas pela soma das contribuições dos níveis dos fatores (STEENKAMP, 1987).

Os resultados foram analisados de acordo com o modelo "clustering segmentation" (MOORE, 1980). Inicialmente, os dados foram analisados individualmente, ou seja, foram calculados os coeficientes de preferências (CPs) para cada um dos consumidores. A partir dos CPs dos consumidores, estes mesmos consumidores foram agrupados utilizando o método average distance e a distância Euclidiana como medida de

Tabela 3 - Tratamentos avaliados no estudo

\begin{tabular}{cccc}
\hline Tratamentos & Design & Nome & Texto Informativo \\
\hline 1 & Azul com estrelas e & Leite Noturno & com \\
& desenho de leite derramando & & \\
& Azul com estrelas e & Leite Noturno & sem \\
& desenho de leite derramando & & \\
3 & Azul com estrelas e & Melatonina & com \\
4 & desenho de leite derramando & & \\
& Azul com estrelas e & Melatonina & sem \\
5 & desenho de leite derramando & & \\
6 & Branca sem desenho & Leite Noturno & com \\
7 & Branca sem desenho & Leite Noturno & sem \\
8 & Branca sem desenho & Melatonina & com \\
9 & Branca sem desenho & Melatonina & sem \\
10 & Azul com estrelas & Leite Noturno & com \\
11 & Azul com estrelas & Leite Noturno & sem \\
12 & Azul com estrelas & Melatonina & com \\
\hline
\end{tabular}


dissimilaridade (KHATTREE; NALK, 2000). Nesse método, grupos inicialmente constituídos (n grupos) foram reunidos de acordo com a proximidade dos elementos, e então os indivíduos mais próximos foram fundidos. Um indivíduo candidato a um agrupamento apresenta uma distância a este grupo igual à média da distância com relação aos membros do agrupamento, ou seja, o coeficiente de distância entre dois agrupamentos $\mathrm{J}$ e $\mathrm{K}$ é dado por:

$\mathrm{d}_{\mathrm{j}, \mathrm{k}}={\text { média } \mathrm{d}_{j k}}$

$j_{\epsilon} J ; k_{\epsilon} K$

Os grupos foram formados por consumidores que apresentaram CPs semelhantes, ou seja, comportamento de compra semelhante. Foram realizadas análises agregadas para cada grupo formado, estimando-se os CPs para cada um.

Os CPs foram estimados por meio de análise de regressão linear múltipla com dummies variáveis, utilizando o método de mínimos quadrados.

Todas as análises estatísticas foram feitas utilizando o programa SAS (Statistical Analysis System - SAS Institute Inc., North Carolina, USA 1999).

\section{RESULTADOS E DISCUSSÃO}

\section{Opiniões e atitudes dos consumidores em} relação ao rótulo de leite

A Figura 3 mostra o perfil dos participantes do grupo de focos. Do total de participantes, $67,5 \%$ eram do sexo feminino e tinham idade entre 22 e 80 anos, sendo que $68 \%$ apresentavam idade superior a 52 anos. Em relação ao grau de instrução, somente $11,2 \%$ tinha apenas o curso superior completo e $45 \%$, segundo grau completo. Quanto à renda familiar, a maioria $(45,6 \%)$ tinha renda de 1 a 5 salários mínimos e somente $9 \%$ deles tinham renda superior a 10 salários mínimos.
Em relação à frequência de consumo, $85 \%$ dos entrevistados consumiam leite todos os dias e somente $7 \%$ consumiam somente 1 vez por semana.

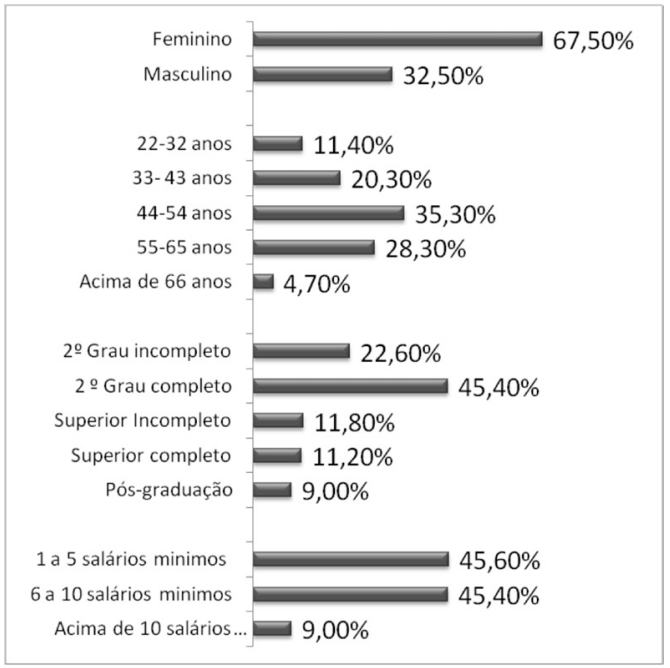

Figura 3 - Perfil dos consumidores que participaram do grupo de focos.

Entre os entrevistados, $49 \%$ disseram ler o rótulo sempre, $46 \%$ disseram ler o rótulo eventualmente e $5 \%$ responderam nunca ler o rótulo (Figura 4).

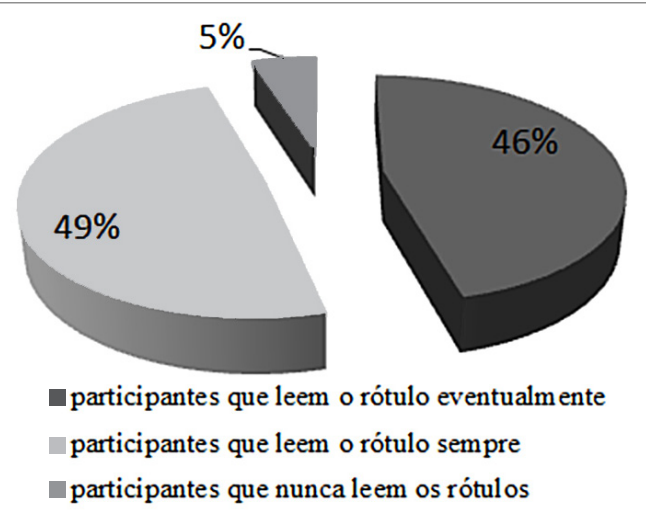

Figura 4 - Frequência de leitura dos rótulos de produtos alimentícios pelos participantes do grupo de focos. 
Observou-se que $32 \%$ dos entrevistados tinham conhecimentos básicos sobre a melatonina obtidos por meio de reportagens de televisão. Porém, a maioria dos entrevistados, $68 \%$, não tinha nenhuma informação sobre a melatonina.

A aparência, a data de validade visível, o preço, a marca, a praticidade e a facilidade de achar a informação que procuram são alguns dos fatores das embalagens de leite que os participantes observam no momento da compra.

Observou-se entre os participantes que a aparência da embalagem é um fator importante na decisão de compra de leite, e $83 \%$ dos entrevistados disseram escolher a embalagem pela aparência. Outro fator considerado importante para os participantes foram informações sobre os ingredientes, e $56 \%$ disseram que observam as informações sobre ingredientes presentes no produto. Os participantes disseram também observar a presença de ingredientes adicionais que melhorem a qualidade nutricional do produto, ou tragam algum beneficio a saúde, sendo que $78 \%$ dos participantes disseram escolher estes produtos.

Entre os fatores de aparência dos produtos, os participantes destacaram o tamanho e as cores das letras, formato da caixa e tipo de tampas, as cores das embalagens, desenhos, cores e quantidade de informação contida nas embalagens e nome do produto.

Todos os entrevistados disseram preferir informações em letras grandes, cores fortes e fáceis de ler. Dos participantes, 92\% disseram escolher embalagens com tampas rosqueáveis e formato não tradicional de caixa de leite UHT. Também preferem o formato mais quadrado e longo pela praticidade de servir e armazenar em geladeira. Sendo assim, foram padronizadas para estudos posteriores as embalagens com letras grandes e fáceis de ler que atendam à Instrução Normativa no 22 (BRASIL, 2005). A tampa rosqueável e o formato não tradicional da caixa UHT também foram padronizados, uma vez que atendem aos desejos da quase totalidade dos participantes.

Quanto ao fator cor das embalagens, $42 \%$ disseram preferir embalagens brancas, pois remetem melhor ao produto leite. Porém, $58 \%$ responderam escolher embalagens coloridas, que chamam mais atenção na prateleira.

Dos entrevistados, 32\% preferem embalagens sem desenhos, com um visual mais limpo, pois permitem visualizar melhor as informações. Já $28 \%$ deles têm preferência por embalagens com desenhos de leite derramando, que estimulam a vontade beber leite ("dar vontade de beber leite"); e 40\% gostam de embalagens com desenho de fazenda e vacas, pois remetem a um produto natural.

Porém, segundo a Instrução Normativa $n \circ 22\left(\mathrm{IN} \mathrm{n}^{\circ} 22\right)$ (BRASIL, 2005), os produtos de origem animal embalados não devem ser descritos ou apresentar no rótulo vocábulos, sinais, denominações, símbolos, emblemas, ilustrações ou outras representações gráficas que possam tornar as informações falsas, incorretas, insuficientes, ou que possam induzir o consumidor a equívoco, erro, confusão ou engano em relação à verdadeira natureza, composição, procedência, tipo, qualidade, quantidade, validade, rendimento ou forma de uso do produto de origem animal. Desta forma é vedado pela IN n॰ 22 (BRASIL, 2005), o uso de fazendas, vacas e imagens que possam induzir ao consumidor que o produto é natural.

Quando questionados a respeito do nome do produto, $62 \%$ aprovaram o nome leite noturno, porém $48 \%$ disseram preferir um nome contendo melatonina.

Pelos dados levantados no grupo de focos, foi possível definir os níveis e fatores para o estudo da avaliação de intenção de compras das embalagens de leite com alta concentração de melatonina e também alguns atributos considerados importantes para a maioria dos participantes. 
Avaliação da intenção de compra das embalagens por meio de Análise Conjunta de Fatores

Por meio do modelo de análise individual, os Coeficientes de Preferências (CPs) foram estimados para cada consumidor e, posteriormente, foi feita a análise de agrupamento. A relação entre o número de grupos formados durante essa análise e o número de julgadores presentes em cada um dos grupos está apresentada na Tabela 4 .

Para análise agregada, considerou-se o agrupamento com seis grupos. Desses seis grupos, 3 eram constituídos de 1 ou 2 julgadores, não sendo, portanto, discutidos. As análises dos resultados foram então feitas para três grupos. A Tabela 5 mostra os resultados da análise agregada para cada grupo formando.

Tabela 4 - Resumo dos resultados de análise de agrupamentos

\begin{tabular}{ccccccccc}
\hline \multicolumn{1}{c}{ Número de Consumidores por Grupo } \\
\hline Número de grupos & 1 & 2 & 3 & 4 & 5 & 6 & 7 & 8 \\
\hline 1 & 144 & & & & & & & \\
2 & 141 & 3 & & & & & & \\
3 & 141 & 2 & 1 & & & & & \\
4 & 106 & 35 & 2 & 1 & & & & \\
5 & 105 & 35 & 2 & 1 & 1 & & & \\
6 & 95 & 35 & 11 & 2 & 1 & 1 & & \\
7 & 94 & 34 & 11 & 2 & 1 & 1 & 1 & \\
8 & 85 & 34 & 11 & 9 & 2 & 1 & 1 & \\
\hline
\end{tabular}

Tabela 5 - Resumo da análise agregada conjunta de fatores, considerando 3 grupos formados

\begin{tabular}{lccc}
\hline \multicolumn{1}{c}{$\begin{array}{c}\text { Fatores e Níveis/ } \\
\text { Importância Relativa }\end{array}$} & $\begin{array}{c}\text { Grupo 1 } \\
(\mathrm{n}=95)\end{array}$ & $\begin{array}{c}\text { Grupo 2 } \\
(\mathrm{n}=35)\end{array}$ & $\begin{array}{r}\text { Grupo 3 } \\
(\mathrm{n}=11)\end{array}$ \\
\hline $\begin{array}{l}\text { Design da embalagem } \\
\text { 1.Azul com estrelas e desenho de }\end{array}$ & 0,8579 & $-0,6090$ & 2,1470 \\
leite derramando. & & & \\
2.Branca sem desenho. & $-1,0142$ & 1,3517 & $-3,1712$ \\
3.Azul com estrelas e sem desenho. & 0,1563 & $-0,7426$ & 1,0242 \\
Importância Relativa & $58,56 \%$ & $66,09 \%$ & $91,69 \%$ \\
Nome & & & \\
1. Leite Noturno & 0,3538 & 0,3986 & $-0,1159$ \\
2. Melatonina & $-0,3538$ & $-0,3986$ & 0,1159 \\
Importância Relativa. & $22,13 \%$ & $25,15 \%$ & $3,99 \%$ \\
Texto Informativo & & & \\
1.Com texto & 0,3084 & 0,1386 & 0,1250 \\
2. Sem Texto & $-0,3084$ & $-0,1386$ & $-0,1250$ \\
Importância Relativa. & $19,29 \%$ & $8,74 \%$ & $4,31 \%$ \\
\hline
\end{tabular}


O primeiro grupo, representado por $66 \%$ dos consumidores, teve sua intenção de compra baseada em todos os fatores, tendo o design da embalagem obtido maior importância relativa ( $I R=58,56 \%)$, seguido do nome $(22,23 \%)$ e do texto informativo (19,29\%). A embalagem ideal para este grupo seria a azul com estrelas e desenho de leite derramando, com o nome leite noturno e com a presença de texto informativo.

Os sinais negativos para os níveis de cada fator indicam impacto negativo na intenção de compra. Considerados importantes para o grupo, apenas fatores com importância relativa acima de 10\% (MINIM, 2010).

$\mathrm{O}$ segundo grupo, compreendendo $24,3 \%$ dos consumidores, teve sua intenção de compra baseada no design da embalagem (IR $=66,09 \%)$. Nesse grupo, diferentemente dos demais, a embalagem branca sem desenhos foi a que obteve maior intenção de compra. O texto informativo apresentou importância relativa menor que $10 \%$, não sendo considerado fator importante. Para esse grupo, a embalagem ideal seria branca, sem desenhos, com o nome leite noturno.

O terceiro grupo, formado por $9,7 \%$ dos consumidores, teve sua intenção de compra baseada no design da embalagem (IR = $91,69 \%$ ), sendo que os demais fatores tiveram importância relativa menor que $10 \%$, não sendo considerados fatores de importância. A embalagem ideal para esse grupo seria a azul com estrelas e desenho de leite derramando.

Desta forma, a embalagem azul com estrelas e desenho de leite derramando, com o nome leite noturno e com a presença de texto informativo (Figura 5), apresentou alta intenção de compra para a maioria dos consumidores.

\section{CONCLUSÕES}

Conclui-se neste trabalho que fatores como nome, design de embalagem, e informação são fatores considerados importantes pelos consumidores de leite. Porém, para os três grupos formados, o fator design da embalagem teve maior importância relativa que nome ou texto informativo.

Desta forma, observa-se que apesar do aumento da tendência dos consumidores lerem os rótulos, esses ainda escolhem o produto baseado no design da embalagem. Embalagem mais coloridas, com formatos mais modernos, e que se diferenciam das demais na prateleira ainda são fatores que os consumidores consideram na compra do produto.

O texto informativo foi o fator de menor importância relativa em todos os grupos, o que demonstra que os consumidores não dão importância as informações contidas na embalagem.

A embalagem com maior intenção de compra para o produto desenvolvido foi a de cor azul, com estrelas e leite derramando, com o nome leite noturno e presença de texto informativo.

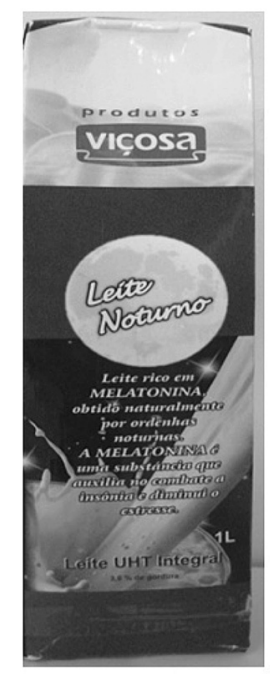

A

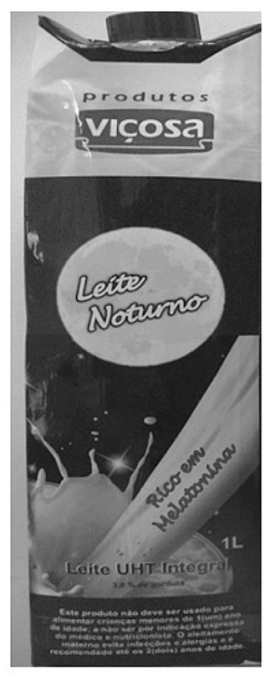

B
Figura 5 - Embalagem com maior intenção de compra: a- verso e b- frente. 


\section{REFERÊNCIAS}

BRASIL. Ministério da Agricultura Pecuária e Abastecimento. Instrução Normativa $n^{\circ}$ 22, de 24 de novembro de 2005. Aprova o Regulamento Técnico para Rotulagem de Produto de Origem Animal Embalado. Diário Oficial da República Federativa do Brasil, Brasília, 25 nov. 2005. Seção 1, p. 15.

CARNEIRO, J. D. S. et al. Labelling effects on consumer intention to purchase for soybean oil.Food Quality and Preference, v. 16, n.3, p. 275-282, 2005.

COSTA, M. C. Tecnologias não convencionais e o impacto no comportamento do consumidor. 1999. 119p. Dissertação (Mestrado em Ciência e Tecnologia de Alimentos) - Universidade Federal Rural do Rio de Janeiro, Rio de Janeiro, 1999.

DELLA LUCIA, S. M. et al. Fatores da embalagem de café orgânico torrado e moído na intenção de compra do consumidor. Ciência e Tecnologia de Alimentos, v. 27, n.3, p. 485-491, 2007.

DELLA LUCIA, S. M.; MINIM, V. P. R. Grupo de foco. In: MINIM, V. P. R. (Ed.). Análise sensorial: estudos com consumidores. Viçosa: UFV, 2010. cap. 4, p. 83-107

FERRAREZI, A. C. Interpretação do consumidor, avaliação da intenção de compra e das características físico-químicas do néctar e do suco de laranja pronto para beber. 2008. 104f. Dissertação (Mestrado em Ciência dos Alimentos) - Universidade Estadual Paulista "Júlio de Mesquita Filho", Araraquara, 2008.

FRATA, M. T. et al.Atributos da embalagem e intenção de compra de suco e néctar de laranja. Semina: Ciências Agrárias, v.30, n.4, p. 847-858, 2009.
GADIOLI, I. L. et al. Evaluation of packing attributes of orange juice on consumers' intention to purchase by conjoint analysis and consumer attitudes expectation. Journal of Sensory Studies, v.28, n.1, p.57-65, 2013.

GONÇALVES, A.C.A. Desenvolvimento de bebida a base de café adicionada de concentrado proteico de soro: da pesquisa mercadológica a avaliação sensorial. 2009. 133f. Dissertação (Doutorado em Ciências de Alimentos) - Universidade Federal de Viçosa, Viçosa, 2009.

GREEN, P. E.; SRINIVASAN, V. Conjoint Analysis in consumer research: Issues and outlook. Journal of Consumer Research, v.5, n. 2, p.103-123, 1978

HAIGH, B. S. Method for producing milk with an enhanced content of naturally expressed melatonin. UK PATENT APLICATION n. 2387009 A, 08 out.2003.

KENNAWAY, D. J.; WRIGHT, H. Melatonin and Circadian Rhythms, Current Topics in Medicinal Chemistry, v.2, n.2, p.199-209, 2002.

KHATTREE, R.; NAIK, D.N. Cluster Analysis.In: Multivariate data reduction and discrimination with SAS Software. Cary NC: SAS Institute Inc, 2000, Cap.6, p. 347-442.

MACFIE, H.J.et al. Designs to balance the effect of order of presentation and first-order carry-over effects in halls tests. Journal of Sensory Studies, v.4, n.2, p.129-148, 1989.

MESIAS, F. J. et al. Evaluation of New Packaging Formats for Dry-Cured Meat Products Using Conjoint Analysis: An Application to Dry-Cured Iberian Ham. Journal of Sensory Studies, v.28, n.3, p. 238-247, 2013. 
Milagres, M.P. Leite de Vaca com Concentração Aumentada de Melatonina: Obtenção, Avaliação Sensorial e Biológica. 2012. 105f. Tese (Doutorado em Ciência dos Alimentos) -Universidade Federal de Viçosa, Viçosa, 2012.

MINIM, V. P. R. Análise Sensorial: estudo com consumidores. 2 ed. Viçosa: Editora da Universidade Federal de Viçosa, 2010. 308p.

MOORE, W. L. Levels of aggregation in conjoint analysis: an empirical comparison. Journal of Marketing Research, v.17, n.4, p.516-523, 1980.

SOUZA, E.A.M et al. Modeling consumer intention to purchase fresh produce. Journal of Sensory Studies, v.22, n.2, p.115-125, 2007.

STEENKAMP, J-B.E. M. Conjoint measurement in ham quality evaluation. Journal Agricultural Economics, v.38, n.3, p.473-480, 1987.

SWEERE, Y. et al. The validity of the Dutch Sleep Disorders Questionnaire (SDQ). Journal of Psychosomatic Research, v.45, n.6, p.549-555, 1998.

VALTONEN, M.; KANGAS, A. P.; VOUTILAINEN, M. Method for producing melatonin rich milk. Patent Cooperation Treatyn. WO2001001784 A1, 11 jan. 2001. 